Edited by Kiriakos Xenitidis and
Colin Campbell
Contents
- Is the moon there when nobody looks?
- Suicide in Wales
Clinimetrics and psychometrics: never the twain

\section{Is the moon there when nobody looks?}

The title of this letter is a comment made by Einstein to one of his assistants in relation to the hotly debated interpretation of quantum mechanics. At the heart of this debate is a seemingly simple statement: in the quantum world, objective states do not exist prior to an act of measurement. So, in a sense, the act of measurement is an integral part of phenomenology itself. The problem, though, is what does an act of measurement entail? Put differently, where does an act of measurement start and/or end? In the world of physics, it is still debated as to what an act of measurement is. The deterministic Schrödinger wave equation mysteriously collapses into specific probability numbers once subject to an act of measurement. For people who are puzzled by the relationship between quantum theory and psychiatric disorders, they should look no further than the fact that the identification of the phenomenology of psychiatric disorders is subject to almost the same conceptual difficulties. For example, any serious attempt to identify the aetiology of depression in a society will inevitably require knowledge of prevalence and incidence rates, which in turn requires the employment of certain diagnostic criteria that assume the existence of depression as an entity in the first place (a priori). So, in a way, the argument is partly circular. Put differently, depression as a 'disorder' cannot claim a separate existence to that of 'diagnostic processes' via the use of measuring tools. Therefore, in the process of trying to identify the phenomenology of mental disorders, the question is: 'What does an act of measurement entail?' In daily practice, a mental health professional's act of measurement revolves around the application of widely used international criteria: the ICD and DSM classification systems. But where do these classification systems come from? The answer is that both ICD and DSM systems have been produced by almost a century of amalgamation of clinical experience, consensus, philosophical schools, political influences and even World Wars. Therefore, current diagnostic systems represent historical processes with a particular narrative. The classification system's historical narrative assumes more relevance to the identification of mental health phenomenology than patients' personal narratives. Mental health phenomenology would not have existed without this historical narrative of our current diagnostic schemes. No wonder it has proved so difficult to reduce mental health phenomenology into basic blocks that can be reliably identified, considering the hundreds of variables embedded into the historical development of our classification systems.

Every time we measure the phenomenology of mental disorders we end up invoking the whole historical development of our classification systems. There is simply no line of demarcation that can separate phenomenology of mental disorders from that of the measurement process in the course of making a diagnosis. It is customary nowadays to describe the current classification systems as reliable, partly because they permit clinicians to communicate using the same language. I think this is a fallacy. Diagnostic systems are inherently operational, i.e. they are more about the 'processes' clinicians follow in order to reach a diagnosis. Therefore, it is inconceivable that two clinicians from two different cultures will end up going through exactly the same steps during the process of making a diagnosis, as this is likely to be influenced by personal experience, semantics, cultural issues and so forth. In my opinion, the diagnostic systems we currently have are inherently more valid than we are willing to accept. It is illogical to say that diagnostic systems are not valid enough because they do not accurately represent what is out there. In reality, they create what is out there. It does not even require a 'conscious' observer to do so. We only need to look!

Saad F. Ghalib, consultant old age psychiatrist, Behavioral Sciences Pavilion, Sheikh Khalifa Medical City, Abu Dhabi, United Arab Emirates. Email:

saadghalib@yahoo.co.uk

doi: 10.1192/bjp.201.2.159

\section{Suicide in Wales}

The paper by Mok et al ${ }^{1}$ makes for interesting reading but is something of a blunt instrument in the presentation of the figures.

By combining figures for England and Wales it overlooks the fact that the suicide rate for men in Wales is significantly higher than in England, with a peak between 20 and 39 years of age. For women in Wales the rate is a little higher than the English average. Within Wales the rate varies considerably, with higher rates in areas of high social deprivation. ${ }^{2}$

The figures for Wales are not as high as those for Scotland and Northern Ireland but have been of sufficient concern for the Welsh Government to launch its suicide prevention campaign 'Talk to $\mathrm{Me}^{33}$ in 2008, which is full of good intentions but lacks some detail as to real-life implementation. The impact of this is yet to be evaluated but it is not likely to have been helped by recent economic problems. The association between social adversity and suicide is easy to identify but harder to change.

Also of interest is the high incidence of drowning in Scotland. Of note is the fact that Scotland accounts for $90 \%$ of the standing freshwater of Great Britain and water makes up around 2\% of the land area of Scotland compared with $0.5 \%$ in England. ${ }^{4}$ Similarly in Ireland, drowning accounts for approximately a fifth of male suicides. ${ }^{5}$ Although it may be simplistic to equate methods of suicide with geographical proximity, it is interesting to speculate on an individual's relationship with their culture, landscape and history which may have a bearing on attitudes to suicide and methods chosen. Drowning, for example, could be more easily explained away as accidental in cultures where everyday use of water for recreation or work is more commonplace, thereby avoiding a pronouncement of suicide and its associated stigma.

Although there are some common, well-replicated associations with completed suicide as discussed in Mok et al, broad statistics tend to hide subtle variations within regions and it is examination of these differences that can inform suicide prevention strategies that are relevant, practical, acceptable and beneficial for a given community.

1 Mok PLH, Kapur N, Windfuhr K, Leyland AH, Appleby L, Platt S, et al. Trends in national suicide rates for Scotland and for England \& Wales, 1960-2008. Br J Psychiatry 2012; 200: 245-51.

2 National Public Health Service for Wales. Suicide in Wales: Data to Support Implementation of the National Action Plan to Reduce Suicide and Self Harm in Wales. Welsh Assembly Government, 2008. 
3 Welsh Assembly Government. Talk to Me: A National Action Plan to Reduce Suicide and Self Harm in Wales 2008-2013. Welsh Assembly Government, 2008

4 Scottish National Heritage. National Heritage Zones. A National Assessment of Scotland's Fresh Water. Scottish National Heritage, 2001.

5 Connelly J. Suicide by drowning in Ireland. World Conference on Drowning Prevention, 2007 (http://www.ilsf.org/nl/node/1733).

Timothy N. Evans, consultant psychiatrist, Cefn Coed Hospital, Swansea, Wales. Email: tnigelevans@talktalk.net

doi: 10.1192/bjp.201.2.159a

Authors' reply: Our rationale for investigating suicides in England \& Wales jointly was twofold. First, although the male suicide rate has been somewhat higher in Wales than in England in recent years, the difference was not nearly as marked as that for Scotland $v$. England, as shown in Fig.1. The pattern of change in trends over the past two decades was also more similar for England $v$. Wales than when compared with Scotland. During the same period, there was little difference in the female suicide rate between England and Wales, while the female rate in Scotland was significantly higher than in the other two countries.

Our second reason was a technical one. At the beginning of our study, we sought advice from the General Register Office for Scotland and the Office for National Statistics about the comparability of their routinely reported suicide data. Because of differences in how suicide data were typically extracted by the two organisations, based on the usual residence and place of death of the deceased, it was concluded that using data from England \& Wales combined would give us the best comparison with the Scottish data.

The cultural and ascertainment explanations of higher rates of suicide by drowning in Scotland proposed by Evans are interesting and plausible. An investigation of suicides in Scotland by Platt et al has reported that drowning as a method of suicide is more common in the Highlands and the Islands than other local areas in the country. ${ }^{1}$ This method of suicide, however, would not have accounted for much of the overall differential between Scotland and England \& Wales, for two reasons. First, it is a relatively rare method, accounting for $5 \%$ of all suicide cases in England \& Wales, and 10\% of those in Scotland, between 2000 and 2008,

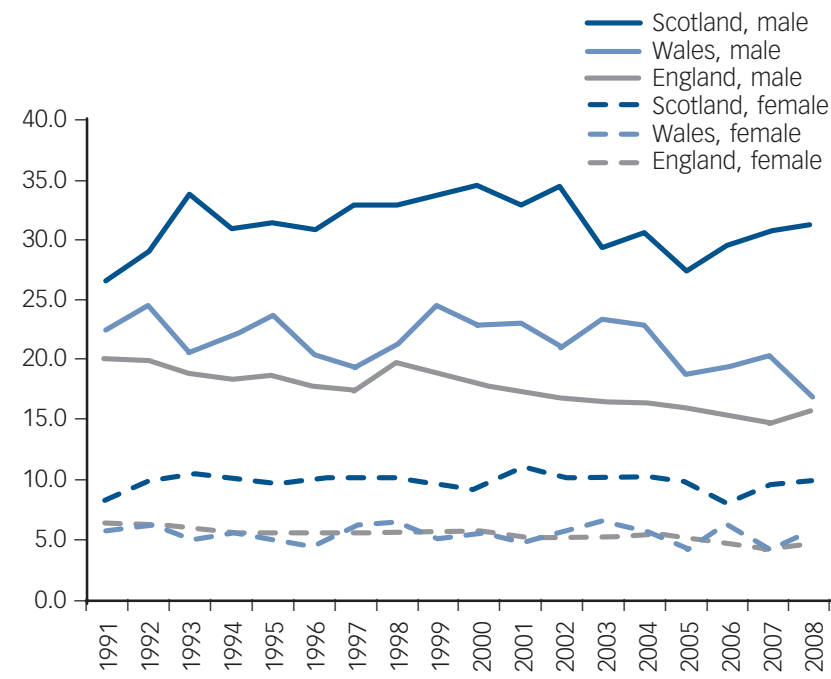

Fig. 1 Age standardised suicide rates by country, 1991-2008 (persons aged 15 and over). Data from General Register Office for Scotland and the Office for National Statistics. and therefore would have made only a minor contribution to the overall between-country differential in risk. Second, although Scotland may contain the great bulk of all the standing water in Great Britain, most Scottish people who died by suicide lived in large urban areas located a considerable distance from the Highlands, where access to such a suicide method was unlikely to be any greater than was the case in England \& Wales.

\section{Declaration of interest}

L.A. is the National Clinical Director for Health and Criminal Justice, and chairs the Suicide Prevention Advisory Group at the Department of Health. He was also the National Director for Mental Health in England between 2000 and 2010.

1 Platt S, Boyle P, Crombie I, Feng Z, Exeter D. The Epidemiology of Suicide in Scotland 1989-2004: An Examination of Temporal Trends and Risk Factors at National and Local Levels. Scottish Executive Social Research, 2007.

Pearl L. H. Mok, Navneet Kapur, Kirsten Windfuhr, Louis Appleby, Centre for Suicide Prevention, University of Manchester, Manchester Academic Health Science Centre; Alastair H. Leyland, Medical Research Council/Chief Scientist Office Social and Public Health Sciences Unit, Glasgow; Stephen Platt, Centre for Population Health Sciences, University of Edinburgh; Roger T. Webb, Centre for Suicide Prevention, University of Manchester, Room 2.311, Jean McFarlane Building, Oxford Road, Manchester M13 9PL, UK. Email: roger.webb@manchester.ac.uk

doi: 10.1192/bjp.201.2.160

\section{Clinimetrics and psychometrics: never the twain shall meet?}

Meta-analysis is upheld as a higher order analysis but it is not without fault as is evident in the conundrums raised by Hegeman et al's article. ${ }^{1}$ Although the methodology was rigorously applied, even the final list of 11 studies included in the meta-analysis were not entirely comparable, particularly in the area of medical comorbidity, a point highlighted by the authors. Our own clinical experience and findings (possibly shared by other psychiatrists) have been that the elderly do have significant medical comorbidity that affect clinical presentations. In one study, all but one elderly patient had a medical condition and $60 \%$ had two medical conditions. ${ }^{2}$ The meta-analysis also does not take into account the significant role socioeconomic and cultural factors have in depressive symptom development and progression in the elderly. Socioeconomic issues play an important part given the changes in occupation, lifestyle and other roles in the elderly. This paper also serves to highlight the recently resurfaced distinction between clinimetrics and psychometrics. ${ }^{3}$ The relevance and applicability of psychometrically driven research is sometimes difficult to translate for the psychiatrist in clinical settings. Clinicians cannot rely entirely on rating instruments to arrive at a diagnosis and to devise management care plans. Clinicians will sieve through the history and presentations in detail and make global judgements on information presented, a process that goes beyond the one- dimensional nature of checklists and rating scales. Fava et al alluded to the 'sophisticated thinking that underlies clinical decisions' and that is a point that deserves consideration even as we review journal articles on research which is largely psychometrically driven to glean benefit for our clinical practice.

1 Hegeman JM, Kok RM, van der Mast RC, Giltay EJ. Phenomenology of depression in older compared with younger adults: meta-analysis. $\mathrm{Br} \mathrm{J}$ Psychiatry 2012; 200: 275-81.

2 Ko SM, Kua EH, Chow MH. Depression of young and elderly patients. Singapore Med J 1997; 38: 439-41. 